The INL is a

U.S. Department of Energy

National Laboratory

operated by

Battelle Energy Alliance

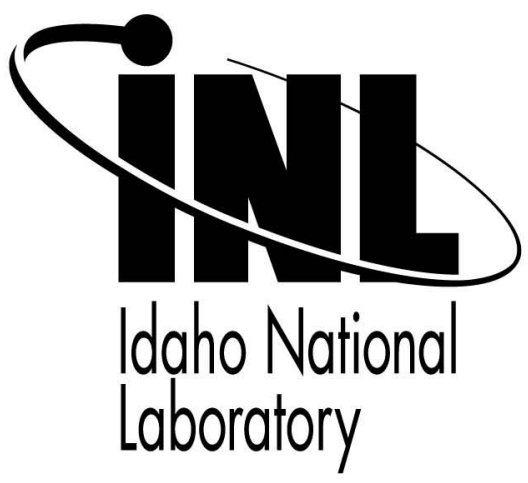

INL/CON-05-00783

PREPRINT

\title{
Aspects of the
}

\section{Fundamental Chemistry \\ of Cesium Extraction \\ From Acidic Media by \\ HCCD}

\section{$15^{\text {th }}$ Radiochemical Conference}

R.S. Herbst

D.R. Peterman

R.D. Tillotson

L.H. Delmau

\section{April 2006}

This is a preprint of a paper intended for publication in a journal or proceedings. Since changes may be made before publication, this preprint should not be cited or reproduced without permission of the author. This document was prepared as an account of work sponsored by an agency of the United States Government. Neither the United States Government nor any agency thereof, or any of their employees, makes any warranty, expressed or implied, or assumes any legal liability or responsibility for any third party's use, or the results of such use, of any information, apparatus, product or process disclosed in this report, or represents that its use by such third party would not infringe privately owned rights. The views expressed in this paper are not necessarily those of the United States Government or the sponsoring agency. 


\title{
ASPECTS OF THE FUNDAMENTAL CHEMISTRY OF CESIUM EXTRACTION FROM ACIDIC MEDIA BY HCCD
}

\author{
R. S. Herbst ${ }^{*}$, D. R. Peterman, R. D. Tillotson \\ Idaho National Laboratory, P. O. Box 1625, Idaho Falls, Id, 83415-2208, U.S.A. \\ L. H. Delmau \\ Oak Ridge National Laboratory, P. O. Box 2008, Oak Ridge, TN, 37831-6119, U.S.A.
}

It is well known that cesium extraction from acidic media by HCCD proceeds through a liquidliquid ion exchange extraction mechanism. Four data sets with 25 experimental measurements of Cs distribution ratios, $\mathrm{D}_{\mathrm{Cs}}=[\mathrm{Cs}]_{\text {org }} /[\mathrm{Cs}]_{\text {aq }}$, at a variety of initial conditions (various concentrations of $[\mathrm{HCCD}]$ and $\left[\mathrm{HNO}_{3}\right]$ ) have been modeled using the SXLSQI computer program developed at ORNL. The SXLSQI program was used in this analysis to help elucidate the general chemical equilibria operative in the extraction of $\mathrm{Cs}^{+}$into an organic phase comprised of HCCD in FS-13. The experimental data sets are best modeled with four chemical equilibria $\left(\mathrm{T}=25^{\circ} \mathrm{C}\right)$. The equilibrium constant for the primary exchange reaction of $\log \mathrm{K}_{\mathrm{eq}}=3.07$ is in excellent agreement with values reported in the literature of $\log \mathrm{K}_{\mathrm{eq}}=3.00$ for the $\mathrm{HCCD} /$ nitrobenzene system. In general, the equilibria representing the mechanism of $\mathrm{Cs}$ extraction by HCCD are consistent with earlier literature reports, albeit derived by different experimental and modeling schemes.

\section{Introduction}

It is now three decades since the initial study of alkali metal extraction by metal bis(dicarbollide) anions was first published [1], and a wealth of excellent scientific information has accumulated related to the extraction chemistry of these unique anions. The hexachlorinated derivative of the cobalt bis(dicarbollide) anion $\left[\left(8,9,12-\mathrm{Cl}_{3}-\mathrm{C}_{2} \mathrm{~B}_{9} \mathrm{H}_{8}\right)_{2}-3-\right.$ $\mathrm{Co}^{-},\left(\mathrm{CCD}^{-}\right)$, typically in the acid form $(\mathrm{HCCD})$, is particularly well known to have a high affinity and selectivity for $\mathrm{Cs}$ in nuclear applications. The early pioneering efforts on dicarbollide chemistry where performed by the Nuclear Research Institute and the Institute of Inorganic Chemistry at Řež, Czech Republic. Numerous collaborative efforts further enhanced the understanding and development of anionic cobalt bis(dicarbollide) with synergistic additives for the simultaneous, efficient recovery of Cs and Sr. These efforts are described and reviewed in the recent literature [2]. Recent collaborations between Russian and US researchers expanded the use of HCCD in the Universal Extraction (UNEX) process. Initially developed for the treatment of acidic aqueous radioactive wastes, the UNEX process simultaneously extracts $\mathrm{Cs}$, Sr, actinides (An), and lanthanides (Ln) by incorporating neutral extractants, diphenyl-N, N-diisobutylcarbamoylmethylphosphine oxide (CMPO) and PEG-400, with HCCD in the diluent phenyltrifluoromethyl sulfone (FS-13) [3-5]. More recently, the experimental work

\footnotetext{
*) c-mail address R.Herbst@inl.gov
} 
focused on understanding the organic phase complexes and structures responsible for $\mathrm{Sr}$ extraction by the synergistic effects of PEG- 400 and HCCD in FS-13 [6]. The current work expands previous studies since FS- 13 is not an extensively studied diluent.

Our experimental observations indicate that multiple chemical equilibria are involved in Cs extraction and activity effects are significant for concentrations greater than $1 \mathrm{M} \mathrm{HNO}_{3}$. This level of complexity requires the use of a computer program, SXLSQI [7-9], which has the capability to calculate quantities such as distribution coefficients, organic phase concentrations, osmotic coefficients, absorbance data, and Karl-Fisher data based on the initial conditions and concentrations of all reagents. The calculated values and experimental data are compared through an agreement factor that reflects the goodness of the fit. Activity coefficients in both organic and aqueous phases are taken into account. The main inputs are the product species assumed formed during extraction. The stoichiometry is fixed by the user and the formation constants for the species are changed automatically by the program to improve the agreement factor. Multiple product species can be considered at the same time.

\section{Experimental}

The Cs salt of chloro-protected cobalt bis(dicarbollide), $\mathrm{Cs}^{+}\left[\left(8,9,12-\mathrm{Cl}_{3}-\mathrm{C}_{2} \mathrm{~B}_{9} \mathrm{H}_{8}\right)_{2}-3-\right.$ $\mathrm{Co}^{-}$(CsCCD) (Katchem, Rež, Czech Republic) was used as received. A concentrated stock solution was prepared by dissolution of the CsCCD salt in FS-13 (Vekton, St. Petersburg, Russia). This stock solution was converted to HCCD by repeated (4-5) batch contacts with equal volumes of $4 \mathrm{M} \mathrm{HClO}_{4}$. This stock solution, nominally at $0.25 \mathrm{M}$ HCCD, was diluted and used in subsequent extraction experiments. The HCCD concentration in the organic phase is determined by potentiometric titration with standard base assuming formation of the $1: 1 \mathrm{H}^{+}$: $\mathrm{CCD}^{-}$complex.

Distribution ratios $\left(\mathrm{D}=[\mathrm{Metal}]_{\mathrm{org}} /[\mathrm{Metal}]_{\mathrm{aq}}\right)$ were measured by equilibrium batch contacts between the organic and aqueous phases at a volume phase ratio of unity $(\mathrm{O} / \mathrm{A}=1)$. The aqueous phase was of the appropriate $\mathrm{HNO}_{3}$ concentration and spiked with ${ }^{137} \mathrm{Cs}$ in trace quantities (typically, less than $10^{-7} \mathrm{M}$ ). In all cases the organic phase was pre-equilibrated by three contacts with fresh $\mathrm{HNO}_{3}$ of the appropriate concentration, followed by contact with the aqueous phase containing traces of ${ }^{137} \mathrm{Cs}$. Phase contact was by vortex mixing for one minute, phase separation by centrifugation, and distribution ratios determined by $\gamma$-spectrometry.

\section{Results and Discussion}

While the physical chemistry of Cs extraction by HCCD is described in the literature and is rather well understood, it cannot be simply explained via slope (log-log) analysis [10]. Indeed, the slopes of the lines representing the dependence of $\mathrm{Cs}^{+}$extraction with nitric acid concentration are steeper than anticipated for simple monovalent cation exchange where one proton is released into the aqueous phase. During the course of our research, numerous values of $\mathrm{D}_{\mathrm{CS}}$ have been experimentally measured as a function of initial $\mathrm{HNO}_{3}$ concentrations for a wide variety of $\mathrm{HCCD}$ containing organic phases. Cesium distributions vs. $\left[\mathrm{HNO}_{3}\right]_{\text {intial }}$ were measured with organic phases comprised of HCCD in conjunction with a variety of 
synergistic extractants such as PEG-400, CMPO, or tetrabutyl-2,6-pyridinedicarboxyl-amide (TBDPA) for the simultaneous extraction of cations such as $\mathrm{Sr}$, An, or Ln. A series of different organic diluents were also studied. A sample of 10 such data sets are indicated by the experimental results shown in Fig. 1 and the associated experimental conditions and results of linear regression $(\log -\log )$ analysis are listed in Table 1.

Table 1. Organic phase compositions and linear regression results from slope analysis.

(Experimental data are presented graphically in Fig. 1)

\begin{tabular}{|c|c|c|c|c|c|}
\hline \multirow{2}{*}{$\begin{array}{c}\text { Data } \\
\text { set }\end{array}$} & \multirow{2}{*}{$\begin{array}{c}{[\mathrm{HCCD}]} \\
(\mathrm{M})\end{array}$} & \multirow{2}{*}{$\begin{array}{c}\text { [Complexant] } \\
\text { (M) }\end{array}$} & \multirow[t]{2}{*}{ Diluent } & \multicolumn{2}{|c|}{ Linear Regression } \\
\hline & & & & Slope & $\begin{array}{c}\text { Correlation, } \mathrm{r}^{2}, \\
\text { (exp. points) }\end{array}$ \\
\hline 1 & 0 & - & FS-13 & +1.10 & 0.9999 (3) \\
\hline 2 & 0.1 & - & FS-13 & -1.27 & 0.991 (7) \\
\hline 3 & 0.0001 & - & FS-13 & -1.36 & $0.992(11)$ \\
\hline 4 & 0.06 & - & FS-13 & -1.73 & $0.995(4)$ \\
\hline 5 & 0.059 & - & nitrobenzene & -1.78 & $0.995(4)$ \\
\hline 6 & 0.068 & 0.03 M PEG-400 & nitrobenzene & -1.69 & $0.994(4)$ \\
\hline 7 & 0.06 & 0.03 M PEG-400 & FS-13 & -1.63 & $0.990(4)$ \\
\hline 8 & 0.02 & $\begin{array}{l}0.002 \mathrm{M} \text { PEG-400 } \\
0.01 \mathrm{M} \text { TBDPA }\end{array}$ & FS-13 & -1.48 & 0.971 (7) \\
\hline 9 & 0.05 & $0.05 \mathrm{M}$ TBDPA & FS-13 & -1.46 & $0.971(3)$ \\
\hline 10 & 0.05 & $0.05 \mathrm{M} \mathrm{CMPO}$ & FS-13 & -1.48 & $0.973(3)$ \\
\hline
\end{tabular}

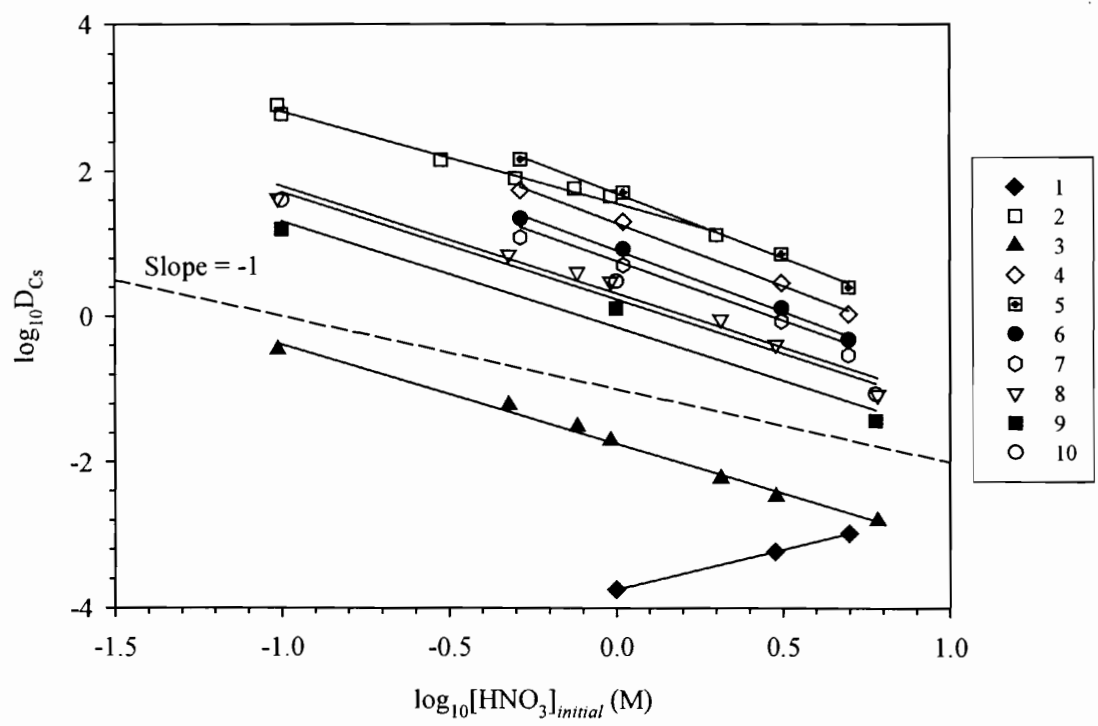

Fig. 1. Cs distribution ratios as a function of the initial nitric acid concentration for a variety of organic phases containing HCCD. Refer to Table 1 for organic phase compositions and results of linear regression analysis. Aqueous phase: $\mathrm{Cs}$ in traces, varying $\left[\mathrm{HNO}_{3}\right] . \mathrm{O} / \mathrm{A}=1$ and $\mathrm{T}=25^{\circ} \mathrm{C}$. 
The primary observation in Fig. 1 and Table 1 is the excellent linearity indicated by the correlation coefficient, $\mathrm{r}^{2},\left(\mathrm{r}^{2}=1\right.$ is a perfect linear fit $)$ and very consistent agreement between the linear slopes for the various data sets. The notable exception is data set 1 , collected for pure FS-13 diluent devoid of HCCD; however, this data set is important for modeling considerations (vide infra). More importantly, for the remaining data sets the slope is always less (or greater in absolute value) than the theoretical value of -1 , and is typically near -1.5. This observed slope of approximately -1.5 has been consistently observed in all of the systems experimentally studied. The objective of this work was to provide additional insight into this unpredicted anomaly of classical slope analysis. Note that only the first four experimental data sets were modeled; these are the only data sets containing only HCCD and FS-13 and are uncomplicated by the addition of other synergistic extractants.

The experimental results in data sets 1 through 4 was modeled with SXLSQI, which uses the Pitzer treatment to account for the non-ideality of the aqueous phase. Hydration of HCCD in the organic phase was also taken into account. Karl-Fisher titrations of the organic phase indicated that the water content remains approximately constant and agree with literature data indicating the hydration number of HCCD is $\sim 5.5$ [11].

In order to model the experimental data, the participation of FS-13 in the overall Cs extraction was first determined by measuring of $\mathrm{D}_{\mathrm{Cs}}$ in the absence of HCCD (data set 1 ). There is a clear dependence $\mathrm{D}_{\mathrm{Cs}}$ with $\mathrm{NO}_{3}{ }^{-}$concentration, suggesting formation of an ion-paired cesium-nitrate complex in the organic phase:

$$
\mathrm{Cs}^{+}+\mathrm{NO}_{3}^{-} \stackrel{\mathrm{K}_{1}}{\longleftrightarrow} \overline{\mathrm{CsNO}_{3}} \quad \log \mathrm{K}_{1}=-3.15 \pm 0.03
$$

It is well known that $\mathrm{Cs}$ extraction in the presence of $\mathrm{HCCD}$ occurs primarily via cation exchange. At high concentrations of $\mathrm{HCCD}$ and $\mathrm{HNO}_{3}$, it is reasonable to expect the species are associated and Cs is extracted as follows:

$$
C s^{+}+\overline{H C C D} \stackrel{\mathrm{K}_{2}}{\longleftrightarrow} \overline{C s C C D}+\mathrm{H}^{+} \quad \log \mathrm{K}_{2}=3.07 \pm 0.09
$$

Note that using only the above two equilibria, the experimental results obtained at higher HCCD concentrations $(0.1 \mathrm{M}$ and $0.06 \mathrm{M} \mathrm{HCCD})$ were adequately modeled while the results obtained at low $(0.0001 \mathrm{M}) \mathrm{HCCD}$ concentration were overestimated (not shown). It was anticipated that at lower HCCD concentrations, dissociation occurs and the following equilibrium should also be included in the model:

$$
C s^{+}+\overline{H^{+}}+\overline{C C D^{-}} \stackrel{\mathrm{K}_{3}}{\longleftrightarrow} \overline{C s^{+}}+\overline{C C D^{-}}+\mathrm{H}^{+} \quad \log \mathrm{K}_{3}=1.54 \pm 0.35
$$

The presence of the $\mathrm{CCD}^{-}$anion is included in (3) to reflect dissociation in the organic phase, and is not included in the expression of $\mathrm{K}_{3}$. Equilibrium 3 indicates the exchange of the HCCD proton for cesium in the organic phase and the following equilibrium indicating the dissociation of HCCD in FS-13 should also be taken into account:

$$
\overline{H C C D} \stackrel{\mathrm{K}_{4}}{\longleftrightarrow} \overline{C C D^{-}}+\overline{\mathrm{H}^{+}} \quad \log \mathrm{K}_{4}=-1.04 \pm 0.35
$$

The results from the SXLSQI model based on equilibria (1)-(4) are compared to the experimental data in Fig. 2. The model is in excellent agreement with each of the experimental data sets. The significant errors on the values of $\mathrm{K}_{3}$ and $\mathrm{K}_{4}$ are primarily due to the uncertainties regarding the hydration number of the different dissociated species. It is 
noteworthy that the calculated value of $\log \mathrm{K}_{2}=3.07$ is in excellent agreement with the mean value reported in the literature of $\log \mathrm{K}=3.00$, albeit derived for the $\mathrm{HCCD}$ /nitrobenzene system at much lower concentrations of HCCD (i.e., dissociated) [12].

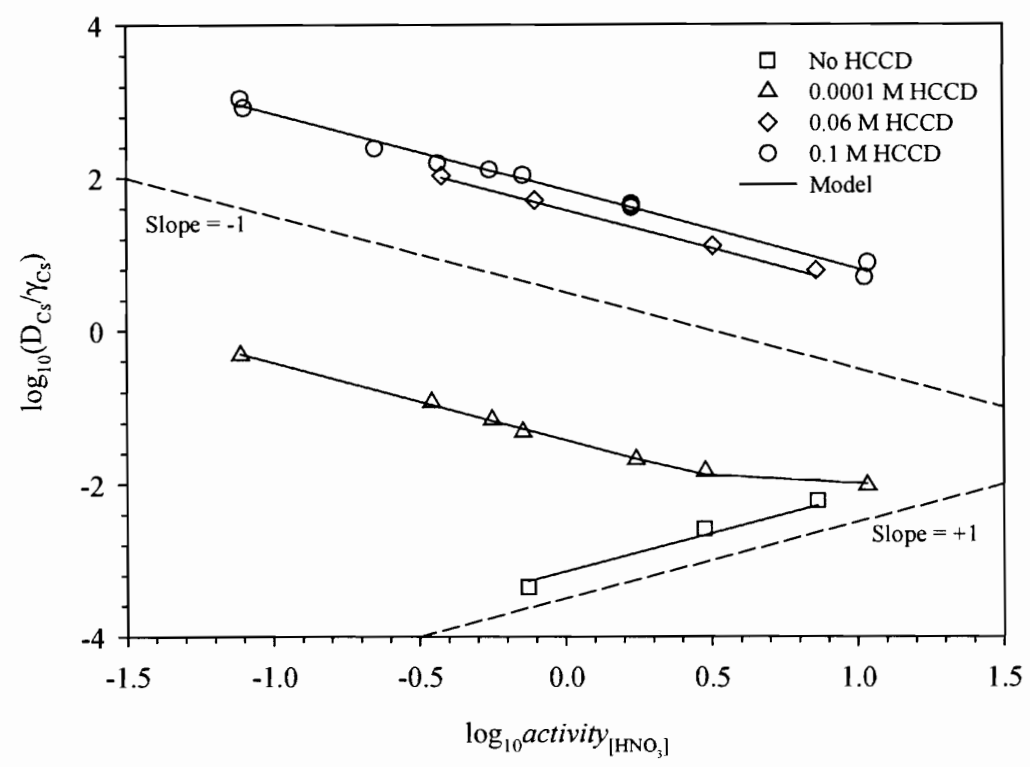

Fig. 2. Cs distribution ratios corrected for the activity of $\mathrm{Cs}$ as a function of the equilibrium activity of $\mathrm{HNO}_{3}$ for different concentrations of $\mathrm{HCCD}$. Aqueous phase: $\mathrm{Cs}$ in traces, varying $\left[\mathrm{HNO}_{3}\right]$. Organic phase: variable $\mathrm{HCCD}$ concentrations in FS-13. $\mathrm{O} / \mathrm{A}=1$ and $\mathrm{T}=25^{\circ} \mathrm{C}$. Model containing ion pairs and dissociated species and plotted to exhibit the influence of activity in the system.

While the experimental data are well described by the above model, it was also necessary to make activity corrections to achieve the expected slope of -1 in the log-log analysis. Assuming that the cation exchange equilibrium (Eq. 2) dominates the overall extraction system, and expressing $\mathrm{K}_{2}$ in terms of activities, $a$, and activity coefficients, $\gamma$, it can be mathematically shown that:

$$
\log \frac{D_{C s}}{\gamma_{C S^{+}}}=-\log a_{H^{+}}+c=-\log a_{N O_{3}^{+}}+c
$$

where the constant, $\mathrm{c}$, is the product of $\mathrm{K}_{2}$ and the molar concentration of [HCCD]. Since SXLSQI calculates activity coefficients for each data point and each ion based on the Pitzer treatment [13], these $\gamma$ values can be conveniently used in the slope analysis.

In addition to activity effects, it is also important to account for the fact that nitric acid is not fully dissociated at high concentrations. The free $\left[\mathrm{H}^{+}\right]$at high nitric acid concentrations will be lower than the total $\left[\mathrm{HNO}_{3}\right]$. This is an important correction; $8 \%$ is associated for an initial concentration of $2 \mathrm{M} \mathrm{HNO}_{3}$ and increases to $16 \%$ for $5 \mathrm{M} \mathrm{HNO}_{3}$. After taking 
into account nitric acid association, correcting the distribution ratios for the activity of cesium, and plotting as a function of the equilibrium nitrate activity as indicated by Eq. (5), the log-log plots in Fig. 2 exhibit -1 slopes for each of the HCCD systems. Also, the system without HCCD (where cesium is extracted as the cesium-nitrate ion pair) exhibits a slope of +1 . It is interesting to note that at the low concentration of $\operatorname{HCCD}\left(10^{-4} \mathrm{M}\right)$ and high concentration of nitric acid $(10 \mathrm{M})$, the distribution ratios start leveling off. This is likely due to the combination of cesium extraction via cation exchange with HCCD and as the cesium nitrate ion pair. This observation further supports the model described above.

\section{Conclusions}

The mechanism of cesium extraction by HCCD in FS-13 was elucidated. Slope analysis cannot be directly applied without a thorough treatment of the data, primarily due to the high ionic strength of the aqueous phase. Activity coefficient corrections demonstrated that slope analysis confirmed the anticipated behavior of the system. Rigorous analysis of the system required the use of the SXLSQI computer program, which takes into account activity effects and confirmed the species formed in the HCCD/FS-13 system. It was determined that the HCCD is present in the organic phase as both the ion paired and dissociated forms. Cesium is likewise extracted with $\mathrm{CCD}^{-}$, either as an ion pair or as a dissociated species. In all cases, the number of water molecules associated with the complexes is in good agreement with reported values. The calculated formation constant for the ion-paired $\mathrm{Cs}^{+} \mathrm{CCD}^{-}$ complex in FS-13 is in very good agreement with literature results for HCCD in nitrobenzene. Understanding the behavior of this system is a solid basis that will be used to study systems where neutral, synergistic extractants are added to extract additional radionuclides.

Acknowledgement: Sponsored by U.S. Department of Energy, under contract DE - AC07-99 ID13727

\section{References}

[1] Rais J., Selucký P. and Kyrš M.: J. Inorg. Nucl. Chem. 38 (7) (1976) 1376.

[2] Rais J. and Gruner B.: Ion Exchange and Solvent Extraction Volume 17, Marcel Dekker New York, NY, (2004) 243.

[3] Herbst R.S. et al.: Sep. Sci. Technol. 37 (8) (1987) 1807.

[4] Herbst R.S. et al.: Solvent Extr. Ion Exch. 20 (4\&5) (2002) 429.

[5] Herbst R.S. et al.: Sep. Sci. Technology 37 (12-13), (2003) 2685.

[6] Luther T. A. et al: J. Radioanal. Nucl. Chem. 267 (3) (2006) 603.

[7] Baes C.F. Jr.: SXLSQI: A Program for Modeling Solvent Extraction Systems, Oak Ridge National Laboratory Report ORNL/TM-13604, December 1998.

[8] Baes C.F. Jr., McDowell W.J., and Bryan S.A.: Solvent Extr. Ion Exch. 5 (1987) 1.

[9] Baes C.F. Jr., Moyer B.A., Case G.N., and Case F.I.: Sep. Sci. Technol. 25 (1990) 1675.

[10] Rais J.: Ion Exchange and Solvent Extraction Volume 17, Marcel Dekker - New York, NY, (2004) 235

[11] Vanura P., Makrlik E., Rais J., and Kyrš M.: Coll. Czech. Chem. Comm. 5(47) (1982) 1444.

[12] Rais J., Selucký P., and Kyrš M.: J. Inorg. Nucl. Chem. 38 (1976) 1376.

[13] Pitzer K. S.: Activity Coefficients in Electrolyte Solutions 2nd ed., CRC Press - Boca Raton, LA (1991). 